\title{
The Interpretation of Some Spheroidal Metrics
}

\author{
W. B. Bonvor and A. SACkFIeld \\ Queen Elizabeth College, London
}

Abstract. ZIPOY recently studied some static gravitational fields in spheroidal coordinatcs, and endowed them with unfamiliar topologies. We examine three of these fields and show that they can also be interpreted as fields in space of Euclidean topology.

\section{$\$ 1$. Introduction}

D. M. Zipoy [7] has presented some static, axially symmetric solutions in spheroidal coordinates of Einstern's equations for empty space

$$
R_{i k}=0 \text {. }
$$

He endowed them with rather terrifying topological properties.

In this paper we examine three of these solutions and show that they can in fact be interpreted quite naturally as gravitational fields in space of Euclidean topology.

\section{The Weyl Solutions}

All solutions studied are members of the Weyl static axially symmetric class [4]. The line element in pseudo cylindrical polar coordinates is

$$
\begin{gathered}
d s^{2}=-e^{2(\lambda-\sigma)}\left(d z^{2}+d \varrho^{2}\right)-\varrho^{2} e^{-2 \sigma} d \phi^{2}+e^{2 \sigma} d t^{2}, \\
(-\infty<z<\infty, 0 \leqq \varrho<\infty, 0 \leqq \phi \leqq 2 \pi,-\infty<t<\infty),
\end{gathered}
$$

where $\lambda$ and $\sigma$ are functions of $z$ and $\varrho$. Following ZIPOY, we transform to pseudo oblate spheroidal coordinates by putting

$$
z=a \sinh u \sin \theta, \quad \varrho=a \cosh u \cos \theta, \quad \phi=\phi, \quad t=t,
$$

$a$ being a constant; (2.1) then becomes

$$
\begin{aligned}
d s^{2}= & -a^{2} e^{2(\lambda-\sigma)}\left(\sinh ^{2} u+\sin ^{2} \theta\right)\left(d u^{2}+d \theta^{2}\right) \\
& -a^{2} e^{-2 \sigma} \cosh ^{2} u \cos ^{2} \theta d \phi^{2}+e^{2 \sigma} d t^{2} .
\end{aligned}
$$

Zipoy takes the ranges of the coordinates as follows

$0 \leqq u<\infty, \quad-\frac{\pi}{2} \leqq \theta \leqq \frac{\pi}{2}, \quad 0 \leqq \phi \leqq 2 \pi, \quad-\infty<t<\infty$.

As is well known, if (2.1) is a solution of (1.1) $\sigma$ has to satisfy LAPLACE's equation; for example in the coordinates of (2.3) $\sigma$ must satisfy

$$
\frac{\partial^{2} \sigma}{\partial u^{2}}+\frac{\partial^{2} \sigma}{\partial \theta^{2}}+\tanh u \frac{\partial \sigma}{\partial u}-\tan \theta \frac{\partial \sigma}{\partial \theta}=0 \text {. }
$$


$\lambda$ is then completely determined up to an additive constant. (2.5) ensures that there is a $1-1$ correspondence between WEYL's solutions and those of Newtonian theory. We shall exploit this fact in the following sections.

\section{\$3. The Monopole Solution}

ZIPOY's monopole solution comes by choosing

$$
\begin{aligned}
& \sigma=-\beta \tan ^{-1}(\operatorname{cosech} u), \quad 0 \leqq \tan ^{-1}(\operatorname{cosech} u) \leqq \pi, \\
& \lambda=\frac{1}{2} \beta^{2} \log \left(\sinh ^{2} u+\sin ^{2} \theta\right)-\beta^{2} \log \cosh u,
\end{aligned}
$$

where $\beta$ is a constant, and where, if $\beta^{2}$ is not integral, the positive value of $e^{2 \lambda}$ is to be taken. As shown by Zipor, this tends to the isotropic Schwarzschild solution as $u$ tends to infinity, the radial coordinate $r$ and the mass $m$ being given by

$$
r=a \sinh u, \quad m=a \beta .
$$

We conclude that (3.1) and (3.2) represent the field of an isolated mass system.

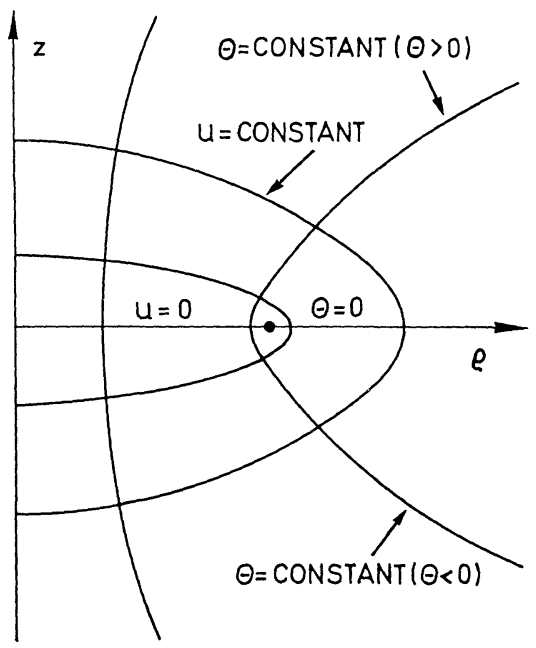

Fig. 1

The ranges for $u$ and $\theta$ being chosen as in (2.4), we must, like ZIPoy, choose the connectivity of $u$ and $\theta$ as shown in Fig. 1. It will be noticed that $\theta$ is discontinuous as lines $\theta=$ const. cross $u=0$. Zipoy calculates the force $f^{1}$ on a stationary unit mass held on the $z$ axis, namely

$$
f^{1}=-\Gamma_{44}^{1}\left(\frac{d t}{d s}\right)^{2}
$$


where $x^{1}=z, x^{4}=t$ refer to metric $(2.1)$. He finds that it suffers a discontinuity as the unit mass crosses $z=0$ because

$$
f_{0+}^{1}=-\frac{\beta e^{-\beta \pi}}{a}, f_{0-}^{1}=+\frac{\beta e^{-\beta \pi}}{a} .
$$

He therefore allows the coordinate $r$ (or $u$ ) to assume the range

$$
-\infty<r<\infty
$$

and introduces a two-sheeted topology to interpret the solution.

However, there is a different, and much simpler, interpretation. Let us take $\sigma$ as the Newtonian gravitational potential in view of (2.5). On $z=0, \varrho \leqq a$ the derivative $\partial \sigma / \partial z$ is discontinuous, and according to elementary potential theory we must take this discontinuity to mean the presence of a surface monopole layer of density $q$ given by

$$
4 \pi q=\left[\left(\begin{array}{c}
\partial \sigma \\
\partial z
\end{array}\right)_{+}-\left(\frac{\partial \sigma}{\partial z}\right)_{-}\right]_{u=0},
$$

taking the gravitational constant as unity. From this we find

$$
q=\beta(2 \pi a \sin \theta)^{-1}, \quad 0 \leqq \theta \leqq \pi / 2 .
$$

We can now check that the source distribution (3.8) gives rise to the Newtonian potential $\sigma$ in (3.1) (see Appendix I). This ensures that there are no other sources, for example, multipole layers which would not be revealed by the discontinuity (3.7).

The Newtonian interpretation of the potential $\sigma$ in $(3.1)$ is therefore that it arises from a disc $\varrho \leqq a, z=0$. We can adopt a similar physical explanation of the relativistic solution (2.3), (2.4), (3.1), and (3.2). The latter has no physical singularities except the disc, and there certain first derivatives of the $g_{i k}$ are discontinuous. We are therefore free to interpret the solution as the field of an isolated monopole disc in space with Euclidean topology.

ISRAEL [3] has recently examined monopole shells and postulates that the second fundamental form is discontinuous across such shells. We shall calculate the second fundamental tensor for our solution. We use metric (2.1) instead of (2.3) because in the latter the normal to the surface $u=0$ is not uniquely defined. We continue to use (2.4), (3.1) and (3.2), regarding $u$ and $\theta$ as parameters in terms of which $\approx$ and $\varrho$ are given by (2.2). The second fundamental tensor $\Omega_{\mu \nu}$ is given by

$$
\Omega_{\mu \nu}=-n_{a ; b} \frac{\partial x^{a}}{\partial v^{\mu}} \frac{\partial x^{b}}{\partial v^{\nu}}
$$

where $a, b$ run from 1 to 4 and $\mu, \nu$ from 2 to $4, n_{a}$ is the unit normal to the disc $S: x^{1} \equiv z=0, x^{2} \equiv \varrho \leqq a$; the semi colon denotes covariant differentiation with respect to the four-dimensional space. The $v^{\mu}$ are 
coordinates on $S$, namely $v^{2}=\varrho, v^{3}=\phi, v^{4}=t$. Then

$$
n_{a}=\left(\left|g^{11}\right|^{-1 / 2}, 0,0,0\right)
$$

and a straightforward calculation leads to the following non-zero components of $\Omega_{\mu \nu}$ on $S$ :

$$
\left(\Omega_{22}, \Omega_{33}, \Omega_{44}\right)=\frac{\beta e^{\frac{1}{2} \beta \pi}}{a \sin \theta \mid(\sin \theta)^{\beta^{2} \mid}}\left(\left|(\sin \theta)^{2 \beta^{2}}\right|, a^{2} \cos ^{2} \theta, e^{-2 \pi \beta}\right) .
$$

Owing to the fact that $\sin \theta$ changes sign as $S$ is crossed these three components are discontinuous, in agreement with the work of IsRael. The discontinuities are finite except at the edge of the disc $(\theta=0)$ where they are infinite.

Following Israte [3] we may introduce the surface energy tensor $S_{\nu}^{\mu}$ by

where

$$
-8 \pi S_{v}^{\mu}=\gamma_{v}^{\mu}-\delta_{v}^{\mu} \gamma_{\alpha}^{\alpha}
$$

$$
\gamma_{\nu}^{\mu}=\Omega_{\nu}^{\mu(-)}-\Omega_{\nu}^{\mu(+)},
$$

and $\Omega_{\nu}^{\mu(-)}, \Omega_{v}^{\mu(+)}$ mean the values of $\Omega_{v}^{\mu}$ as $z \rightarrow 0$ from below or above, indices here being raised by the $g^{\mu \nu}$ in the surface. It then turns out that the only non-zero component of $S_{\nu}^{\mu}$ is

$$
S_{4}^{4}=\frac{1}{2} \beta(a \pi)^{-1}\left|(\operatorname{cosec} \theta)^{\beta^{2}+1}\right| e^{-\beta \pi / 2} .
$$

We may calculate the total mass of the disc by the integral

$$
m=\int_{S} S_{ \pm}^{4} \sqrt{{ }^{(3)}} g d x^{2} d x^{3},
$$

where $S$ is the disc and ${ }^{(3)} g$ is the determinant of the metric on $S$. We find

in agreement with (3.3).

$$
m=\beta a
$$

Rather unexpectedly the stresses $S_{2}^{2}$ and $S_{3}^{3}$ vanish. It turns out, however, that there is a good physical reason for this: in the classical problem of a disc with density (3.8) the material of the disc is in equilibrium under its own gravitation so no stresses are required. (We are indebted to Dr. P. T. SAunders for suggesting this explanation.) Incidentally, it is because the stresses vanish that (3.11) is equal to the total gravitational mass. In the case of a static matter distribution of finite density it is known from WHITTAKER's theorem [5] that the gravitational mass density is $T_{4}^{4}-T_{1}^{1}-T_{2}^{2}-T_{3}^{3}$. For the surface distribution this must be adapted to $S_{4}^{4}-S_{2}^{2}-S_{3}^{3}$; since $S_{2}^{2}$ and $S_{3}^{3}$ vanish this reduces simply to $\mathrm{S}_{4}^{4}$.

One curious fact should be noted. The three invariants of the Riemann tensor, given by ZIPoY, become infinite on the edge $\varrho=a, z=0$, but are bounded near the rest of the disc $\varrho<a-\varepsilon, z=0$. (On the 
disc itself they are not defined because of discontinuities in first derivatives of $g_{i k}$.) This shows that for a physical singularity it is not a necessary condition that these invariants become infinite. It is hoped that this work will act as a corrective to the commonly held belief that the invariants of the Riemann tensor necessarily become infinite at physical singularities. As a further counterexample to this view we may remind the reader that at the apex of an ordinary cone the invariants of the Riemann tensor are perfectly wellbehaved (of course, they vanish). A result supporting our attitude to the Riemann tensor has recently been given by GAUTREAU and ANDERson [2].

\section{$\$ 4$. The Dipole Solution}

ZIPOY also gives a solution consisting of metric (2.3), ranges (2.4) and with

$$
\begin{gathered}
\sigma=\gamma\left[1-\sinh u \tan ^{-1}(\operatorname{cosech} u)\right] \sin \theta, \quad 0 \leqq \tan ^{-1}(\operatorname{cosech} u) \leqq \pi,(4.1) \\
e^{2 \lambda}=\left[\frac{\sinh ^{2} u+\sin ^{2} \theta}{\cosh ^{2} u}\right]^{-\gamma^{2}} \exp \left\{-\sigma^{2} \cot ^{2} \theta-\gamma^{2} \cos ^{2} \theta\left[\tan ^{-1}(\operatorname{cosech} u)\right]^{2}\right\}
\end{gathered}
$$

$\gamma$ being a constant. Introducing $r$ from (3.3) we find that for large $r \sigma$ is the Newtonian potential of a dipole of moment $+\frac{1}{3} \gamma a^{2} \cdot \sigma$ is discontinuous when the disc $z=0, \varrho \leqq a$ is crossed (because $\sin \theta$ is discontinuous) though its first normal derivative is continuous. ZIPOY again introduces a two sheeted topology with $r$ in the range $(3.6)$, which leads him into serious difficulty because his metric is then singular when $r \rightarrow-\infty$.

Once again, however, there is a simple explanation with a classical analogue. The properties of $\sigma$ just mentioned suggest that it is the potential of a sheet of dipoles. Indeed, we find for the magnitude of the discontinuity across the disc $z=0, \varrho \leqq a$,

$$
\sigma_{+}-\sigma_{-}=2 \gamma \sin \theta, \quad 0 \leqq \theta \leqq \pi / 2,
$$

which corresponds to a dipole sheet of strength $(\gamma \sin \theta) / 2 \pi$. As in the case of the monopole disc, we can show that this dipole sheet gives rise to the potential (4.1), so it is the only source present.

There is nothing to stop us adopting a similar interpretation for the relativistic solution (2.3), (2.4), (4.1), (4.2), which we therefore take to refer to a mass dipole sheet on $z=0, \varrho \leqq a$ in space with Euclidean topology. The second fundamental tensor is discontinuous on the disc $z=0, \varrho \leqq a$. Calculations with IsRAEL's surface energy tensor in this case give unsatisfactory results: since the metric on the disc is not uniquely defined, the integral (3.11) is ambiguous. 
ZIPOY gives a solution in which $\sigma$ is the sum of (3.1) and (4.1): $\lambda$ is then the sum of (3.2) and (4.2) with another term added. This too can be interpreted in a space of Euclidean topology: it refers to a superposition of the monopole and dipole discs discussed above, together with a singularity along the axis $0=0, z<0$ which represents a stress holding them in position.

\section{\$. Conclusion}

It is an empirical question whether ZIPOY's solutions represent objects with strange topology, or the simpler objects we have suggested. Those of us who think that the universe is already complicated enough must hope that the latter alternative is the correct one.

\section{Appendix I}

Derivation of potential (3.1) from source distribution (3.8)

Let the matter residing on the disc $z=0, \varrho \leqq a$ have density

$$
q=\beta(2 \pi a \sin \theta)^{-1}=\beta\left\{4 \pi^{2}\left(a^{2}-\varrho^{2}\right)\right\}^{-1 / 2}, \quad 0 \leqq \theta \leqq \pi / 2
$$

from (2.2). On the $z$-axis the Newtonian potential of this distribution is

$$
\Phi=-\int_{S} q \frac{d S}{\sqrt{\varrho^{2}+z^{2}}},
$$

where $d S$ is the element of area and $S$ is the disc. Performing the integration we find

$$
\Phi=-\beta \tan ^{-1}\left(\frac{a}{z}\right) .
$$

It may be shown [1] that if the potential on the $z$-axis is $F(z)$ then the potential off the $z$-axis is:

$$
\Phi=\frac{1}{\pi} \int_{0}^{\pi} F(z+i \varrho \cos \alpha) d \alpha .
$$

From (I.1) we see that in our case

$$
F(z)=-\beta \tan ^{-1}\left(\frac{a}{z}\right) ;
$$

hence from (I.2) the potential off the $z$-axis is

$$
\Phi=\frac{-\beta}{\pi} \int_{0}^{\pi} \tan ^{-1}\left\{\frac{a}{z+i \varrho \cos \alpha}\right\} d \alpha
$$

which may be written

$$
\Phi=\frac{-\beta i}{\pi} \int_{0}^{\pi} Q_{0}\left\{\frac{i(z+i \varrho \cos \alpha)}{a}\right\} d \alpha .
$$


where $Q_{0}$ is the Legendre polynomial of the second kind and zero degree. Transforming $z$ and $\varrho$ to oblate spheroidal coordinates by $(2.2)$ and putting

we have

$$
u=i\left(\frac{\pi}{2}-\eta\right), \quad 0=\frac{\pi}{2}-\xi,
$$

$$
\frac{i(z+i \varrho \cos \alpha)}{a}=-(\cos \eta \cos \xi+\sin \eta \sin \xi \cos \alpha) \text {. }
$$

Hence (I.3) may be written as

$$
\Phi=\frac{\beta i}{\pi} \int_{0}^{\pi} Q_{0}(\cos \eta \cos \xi+\sin \eta \sin \xi \cos \alpha) d \alpha .
$$

Now the addition formula for $Q_{0}$ states that [6]

$Q_{0}(\cos \eta \cos \xi+\sin \eta \sin \xi \cos \alpha)=P_{0}(\cos \xi) Q_{0}(\cos \eta)$

$$
+2 \sum_{m=1}^{\infty} P_{0}^{m}(\cos \xi) Q_{0}^{m}(\cos \eta) \cos m \alpha ;
$$

integrating with respect to $\alpha$ between 0 and $\pi$ we have

using (I.4). Hence from (I.5)

$$
\begin{gathered}
\int_{0}^{\pi} Q_{0}(\cos \eta \cos \xi+\sin \eta \sin \xi \cos \alpha) d \alpha=\pi Q_{0}(\cos \eta) \\
=-\pi Q_{0}(i \sinh u)
\end{gathered}
$$

$$
\Phi=-\beta i Q_{0}(i \sinh u)=-\beta \tan ^{-1}(\operatorname{cosech} u) .
$$

This proves that the monopole disc of surface density (3.8) is the only source for the Newtonian potential (3.1).

\section{References}

1. Ferraro, V. C. A.: Electromagnetic theory. London: Athlone Press, University of London 1962.

2. Gautreau, R., and J. L. Anderson: Physics Letters 25 A, No. 4, 291 (1967).

3. Israel, W.: Il Nuovo Cimento, Serie X, 44, 1 (1966).

4. Synge, J. L.: Relativity - the general theory. Amsterdam: North Holland Publishing Company 1960.

5. Whittaker, E. T.: Proceedings of the Royal Society A. 149, 384 (1935).

6. -, and G. N. WATSON: Modern analysis, p. 329. Cambridge University Press 1952.

7. ZIPOY, D. M.: Journal of Mathematical Physics 7, 1137 (1966).

Prof. W. B. Bonnor

Department of Mathematics

Queen Elizabeth College

(University of London)

Campden Hill Road

London, W. 8, Great Britain 